\title{
Femtosecond laser-assisted cataract surgery in a patient with a Collamer lens
}

\section{Cirugía de catarata asistida con láser de femtosegundo en paciente con lente intraocular fáquico de Collamer}

\author{
Christian R. Fau ${ }^{1 *}$ and Romina G. Muñoz ${ }^{2}$ \\ ${ }^{1}$ Ophthalmological Foundation 2020, Iberoamerican Cochrane Network; ${ }^{2}$ Medical Technology School, Universidad Santo Tomás. Santiago, Chile
}

A 47-year-old female patient with high myopia. More than 10 years ago, Collamer's intraocular phakic refractive surgery was performed on both eyes. On examination, the patient showed in the right eye an anterior cortical cataract, secondary to contact of the phakic lens with the anterior surface of the crystalline lens (C5NOP0), which compromised the visual axis and only allowed a corrected eccentric vision of 0.3 with eyeglasses ${ }^{1}$; her left eye had a vision of 0.05 due to myopic retinopathy with foveal damage.

The patient, after consulting several specialists, decided to have femtosecond laser-assisted cataract surgery (VICTUS Technolas equipment). Given the high risk of presenting an adhesion between the phakic lens and the anterior lens capsule, a capsulorhexis was performed through the phakic lens ${ }^{2}$, then the surgery was completed by performing the following steps: paracentesis with a knife, phakic lens and capsulorhexis removal (capsulorhexis was continuous without areas of adhesions), phacoemulsification and intraocular lens implantation; surgery was completed without incidents.

Performing the capsulorhexis with the laser through the phakic lens generates uncertainty as to whether the results will be as expected ${ }^{3}$ and if the capsulorhexis will be continuous, given the risk of loss of power or precision of the laser due to passing through a material for which it was not designed. Both in this case and in the article by $\mathrm{Li}$, et al. ${ }^{3}$, the implanted phakic lens does not

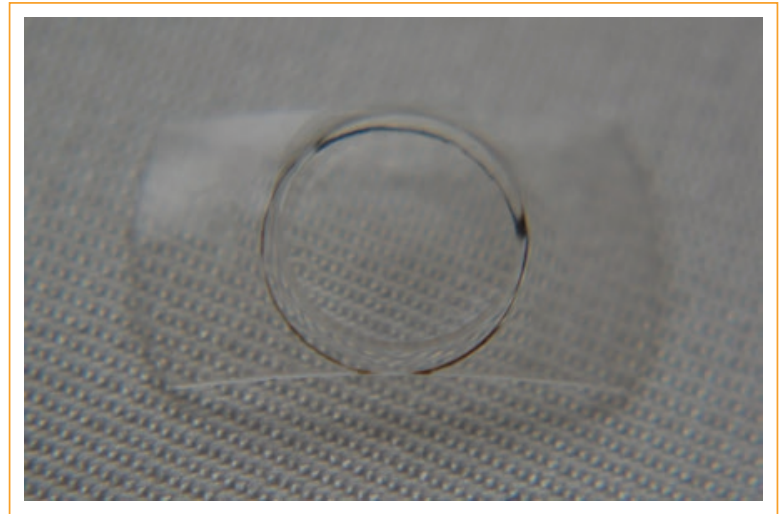

Figure 1. Anterior face of the phakic intraocular lens, a black line generated by the femtosecond laser is observed on the posterior face of the lens.

seem to interfere with the transmission of energy of the femtosecond laser. In this case, the power of the laser not only generated a continuous cut of the capsulorhexis without incidents, but also marked the phakic lens on its inner face, as can be seen in the photographs (Figs. 1 and 2). This demonstrates that the femtosecond laser can perform not only a continuous capsulorhexis through a phakic lens without complications, but also generate a cutting interval, both anterior and posterior to the previously programmed cut point. The mark of the lens does not show that this area extends sideways. 


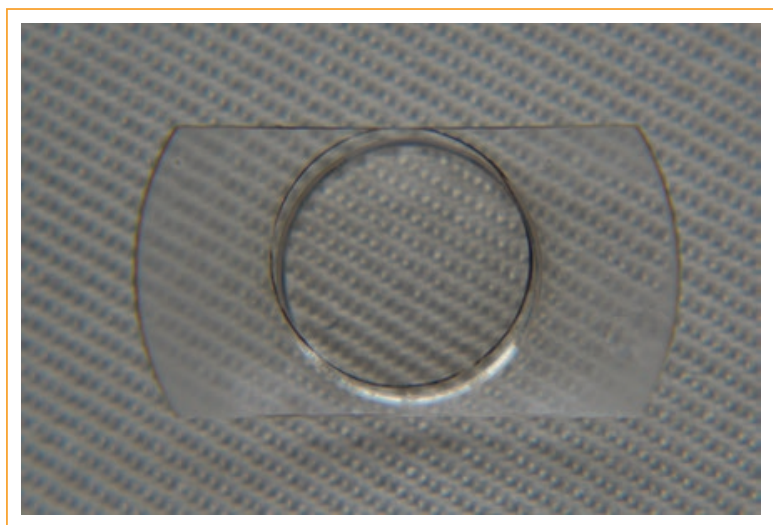

Figure 2. Posterior face of the phakic intraocular lens showing a black cutting line, generated by the femtosecond laser.

This is especially important when cutting the nucleus, since this cutting interval can break the posterior capsule if the fragmentation of the nucleus gets too close.

In conclusion, the use of this method is safe and effective ${ }^{2,3}$, offering surgeons an additional treatment option for these patients, who have high expectations of visual function and often show reduced endothelial cell counts.

Finally, the result of the patient at 6 months after surgery was a corrected visual acuity of 0.8 (partial) and without complications.

\section{Conflicts of interest}

The authors declare no conflict of interests.

\section{Ethical disclosures}

Protection of human and animal subjects. The authors declare that the procedures followed were in accordance with the regulations of the relevant clinical research ethics committee and with those of the Code of Ethics of the World Medical Association (Declaration of Helsinki).

Confidentiality of data. The authors declare that they have followed the protocols of their work center on the publication of patient data.

Right to privacy and informed consent. The authors have obtained the written informed consent of the patients or subjects mentioned in the article. The corresponding author is in possession of this document.

\section{References}

1. Packer M. Meta-analysis and review: Effectiveness, safety, and central port design of the intraocular collamer lens. Clin Ophthalmol. 2016; 10:1059-77.

2. Schultz T, Schwarzenbacher L, Burkhard Dick H. Comparing femtosecond laser-assisted cataract surgery before and after phakic intraocular lens removal. J Refractive Surg. 2018;34(5):343-6.

3. Li S, Chen X, Kang Y, Han N. Femtosecond laser-assisted cataract surgery in a cataractous eye with Implantable Collamer Lens in situ. J Refractive Surg. 2016;32(4):270-2. 